\title{
Results of a prospective study (CATS) on the effects of thalamic stimulation in minimally conscious and vegetative state patients
}

\author{
Lorenzo Magrassi, MD, ${ }^{1}$ Giorgio Maggioni, MD, ${ }^{2}$ Caterina Pistarini, MD, ${ }^{2}$ Carol Di Perri, MD, 3,4 \\ Stefano Bastianello, MD, ${ }^{3}$ Antonio G. Zippo, PhD, ${ }^{5}$ Giorgio A. lotti, MD, ${ }^{6}$ Gabriele E. M. Biella, MD, ${ }^{5}$ \\ and Roberto Imberti, MD ${ }^{6,7}$
}

\begin{abstract}
${ }^{1}$ Neurochirurgia Dipartimento di Scienze Clinico-Chirurgiche, Diagnostiche e Pediatriche, University of Pavia-Fondazione IRCCS Policlinico S. Matteo, Pavia; ${ }^{2}$ Neurorehabilitation Unit, Salvatore Maugeri Foundation IRCCS, Scientific Institute of Pavia; ${ }^{2}$ Neuroradiology Unit, Neurological Institute IRCCS Fondazione C. Mondino, Pavia, Italy; ${ }^{4}$ Coma Science Group, Cyclotoron Research Center, University of Liege, Belgium; ${ }^{5}$ sstituto di Bioimmagini e Fisiologia Molecolare, CNR, Segrate; and ${ }^{6}$ Rianimazione $2^{\circ}$ and ${ }^{7}$ Phase I Clinical Trial Unit and Experimental Therapy, Fondazione IRCCS Policlinico S. Matteo, Pavia, Italy
\end{abstract}

\begin{abstract}
OBJECTIVE Deep brain stimulation of the thalamus was introduced more than 40 years ago with the objective of improving the performance and attention of patients in a vegetative or minimally conscious state. Here, the authors report the results of the Cortical Activation by Thalamic Stimulation (CATS) study, a prospective multiinstitutional study on the effects of bilateral chronic stimulation of the anterior intralaminar thalamic nuclei and adjacent paralaminar regions in patients affected by a disorder of consciousness.

METHODS The authors evaluated the clinical and radiological data of 29 patients in a vegetative state (unresponsive wakefulness syndrome) and 11 in a minimally conscious state that lasted for more than 6 months. Of these patients, 5 were selected for bilateral stereotactic implantation of deep brain stimulating electrodes into their thalamus. A definitive consensus for surgery was obtained for 3 of the selected patients. All 3 patients ( 2 in a vegetative state and 1 in a minimally conscious state) underwent implantation of bilateral thalamic electrodes and submitted to chronic stimulation for a minimum of 18 months and a maximum of 48 months.
\end{abstract}

RESULTS In each case, there was an increase in desynchronization and the power spectrum of electroencephalograms, and improvement in the Coma Recovery Scale-Revised scores was found. Furthermore, the severity of limb spasticity and the number and severity of pathological movements were reduced. However, none of these patients returned to a fully conscious state.

CONCLUSIONS Despite the limited number of patients studied, the authors confirmed that bilateral thalamic stimulation can improve the clinical status of patients affected by a disorder of consciousness, even though this stimulation did not induce persistent, clinically evident conscious behavior in the patients.

Clinical trial registration no.: NCT01027572 (ClinicalTrials.gov)

http://thejns.org/doi/abs/10.3171/2015.7.JNS15700

KEY WORDS vegetative state; unresponsive wakefulness syndrome; minimally conscious state; thalamic stimulation; deep brain stimulation

$\mathrm{P}$ ATIENTS in a vegetative state/unresponsive wakefulness syndrome ${ }^{18}$ (VS/UWS) or in a minimally conscious state ${ }^{8}$ (MCS) are characterized by preserved arousal but behavioral evidence of awareness of self and surroundings are absent (VS/UWS) or inconsistent (MCS).
These conditions are independent of the initial insult that caused the damage to the brain that led the patient to enter these states. Among the patients who remain in a VS/UWS or MCS for at least 4 weeks, approximately half remain in the same condition or, even if they improve, are severe-

ABBREVIATIONS CATS $=$ Cortical Activation by Thalamic Stimulation; CRS-R = Coma Recovery Scale-Revised; DBS $=$ deep brain stimulation; DOC $=$ disorder of consciousness; EEG = electroencephalographic; fMRI = functional MRI; ICA = independent component analysis; IRCCS = Istituto Ricerca Cura Carattere Scientifico; MCS = minimally conscious state; UMRS = Unified Myoclonus Rating Scale; VS/UWS = vegetative state/unresponsive wakefulness syndrome.

SUBMITTED March 31, 2015. ACCEPTED July 20, 2015.

INCLUDE WHEN CITING Published online January 8, 2016; DOI: 10.3171/2015.7.JNS15700. 
ly disabled 1 year later. ${ }^{10}$ Significant recovery in patients with a severe disorder of consciousness (DOC) admitted to an acute inpatient rehabilitation center mostly occurs within the 2 years after the initial injury. ${ }^{23}$ The first clinical study of deep brain stimulation (DBS) with the aim of improving recovery in patients with a DOC dates back to the pioneering research of Hassler et al. ${ }^{13,14}$ in 1969. Their study was conducted in an acute setting, and the patients underwent 15 minutes of daily stimulation of the anterior nucleus of the thalamus and globus pallidus; this stimulation caused some improvements in the patients' level of reactivity to environmental stimuli and in electroencephalographic (EEG) rhythms that lasted after the end of the stimulation. In the following decades, there were many attempts to reproduce and improve the results of Hassler et al. by moving from acute to chronic long-term stimulation with fully implantable DBS devices and by exploring new targets, such as the centromedian-parafascicularis nuclear complex of the thalamus s, $^{3,26,28,31}$ and the mesencephalic reticular formation. ${ }^{28,31}$ Independent of the target of stimulation, the results of these cited studies indicated that DBS induces changes in the EEG pattern, such as an increase in higher-frequency rhythms and desynchronization, which makes the pattern more similar to that of otherwise healthy subjects. ${ }^{5,14,32}$ All the study groups also described clinical improvements, although the results of their studies are difficult to compare because of large differences in the duration of the DOCs, changes in the classification of DOCs with the identification of the MCS, ${ }^{8}$ and multiple developments in implanted hardware over the last 30 years. Despite intense interest in the potential applications of DBS to patients with a DOC, as shown by the publication of several recent reviews of the data available, ${ }^{19,27}$ results of new studies on multiple patients affected by a DOC have not been reported recently.

Here, we report the long-term results of the Cortical Activation by Thalamic Stimulation (CATS) study (clinical trial registration no.: NCT01027572 [ClinicalTrials.gov]), a prospective multiinstitutional study started in 2009 that examined the effects of bilateral DBS of the anterior intralaminar thalamic nuclei and adjacent paralaminar regions of thalamic association nuclei in patients in a VS/UWS or MCS. The primary end point of the study was variation in the level of patient consciousness as indicated by modified Coma Recovery Scale-Revised (CRS-R) $)^{9,15,20}$ scores (Table 1) at least 1 year after bilateral thalamic stimulation of the anterior intralaminar thalamic nuclei and adjacent paralaminar regions. The secondary end point was change in the EEG power spectrum after the same period of bilateral stimulation of the thalamus in the same patients.

\section{Methods}

Patients of both sexes aged between 10 and 65 years affected by a DOC that had lasted more than 6 months were enrolled in the CATS study. The research protocols were approved by the institutional review board of the Fondazione Instituto Ricerca Cura Carattere Scientifico (IRCCS) Policlinico S. Matteo (Pavia, Italy) and later revised and approved by the institutional review boards of the Fondazione IRCCS Maugeri (Pavia) and Fondazione IRCCS
TABLE 1. Six subscales of the CRS-R scale*

\begin{tabular}{lccc}
\hline \multicolumn{1}{c}{ CRS-R Subscale } & $\begin{array}{c}\text { Score } \\
\text { Range }\end{array}$ & $\begin{array}{c}\text { Max VS/UWS } \\
\text { Score }\end{array}$ & $\begin{array}{c}\text { Max MCS } \\
\text { Score }\end{array}$ \\
\hline Auditory function & $0-4$ & 2 & 4 \\
\hline Visual function & $0-5$ & 1 & 5 \\
\hline Motor function & $0-6$ & 2 & 5 \\
\hline Oromotor/verbal function & $0-3$ & 2 & 3 \\
\hline Communication & $0-2$ & 0 & 1 \\
\hline Arousal & $0-3$ & 2 & 3 \\
\hline Total & 23 & 9 & 21 \\
\hline
\end{tabular}

* The lowest score on each subscale indicates purely reflexive activity or the lack thereof, whereas the highest score indicates cognitively mediated behavior. Scoring is based on the presence or absence of specific responses to standardized stimuli organized hierarchically in 29 items. ${ }^{15}$ Total scores $\leq$ 9 are indicative of a VS/UWS unless one or more subscores of the CRS-R subscales exceeds the maximum limits indicated in column 3 (VS/UWS). Total scores $>9$ but $\leq 21$ are indicative of an MCS unless one or more of the subscores of the CRS-R subscales exceeds the maximum limits indicated in column 4 (MCS).

$\dagger$ Range shows scores from minimum to maximum.

Istituto Neurologico Nazionale C. Mondino (Pavia) when they joined the study. Informed consent was obtained for each patient from his or her legal representatives, and evaluation of each patient for enrollment into the study started after request by the person with legal responsibility for the patient. The study was conducted in accordance with the Declaration of Helsinki.

The accepted initial etiologies that led to the patients' DOC were head trauma, spontaneous intracranial hemorrhage, and transient anoxic insult. The level of the DOC affecting the subject was evaluated by using the Italian version of the CRS-R. ${ }^{20}$ Any patient who had no objective indications of a stable DOC noted in the clinical records submitted at the time of the initial request to enter the study was rejected without further consideration.

Inclusion criteria were based on clinical (Table 2), neurophysiological (Table 3), and neuroradiological (Table 4) findings.

Exclusion criteria were the following: further neurological disease independent of the pathology that caused the DOC, a life expectancy of less than 1 year because of extraneurological diseases, an ongoing not-eradicable infection, pregnancy, untreated hydrocephalus, and a request from the legal representative to exclude the patient from the study.

Each patient was independently examined by 2 investigator teams both before and after DBS surgery and at scheduled follow-up controls, which comprised a thorough clinical evaluation including but not limited to the administration of the CRS-R and Sections 2 and 3 of the Unified

TABLE 2. Clinical inclusion criteria

\begin{tabular}{lc}
\hline \multicolumn{1}{c}{ Clinical Parameter } & Criteria \\
\hline Age range (yrs) & $\geq 10$ to $\leq 65$ \\
\hline Duration of stable DOC (mos) & $>6$ \\
\hline CRS-R (Italian version) & $<22^{*}$ \\
\hline
\end{tabular}

* Communication scale maximum 1; motor function scale maximum 5. 
TABLE 3. Neurophysiological inclusion criteria

\begin{tabular}{ll}
\hline \multicolumn{1}{c}{ Investigation } & \multicolumn{1}{c}{ Criteria } \\
\hline Electroencephalography & Desynchronized activity in $<5 \%$ of the total recorded time (continuous electroencephalography $1 \mathrm{hr}$ ) \\
\hline $\begin{array}{l}\text { Somatosensory evoked potentials (median \& } \\
\text { peroneal nerve stimulation) }\end{array}$ & Evoked on $\geq 1$ side (latency of N20 potential may be delayed up to $1 \mathrm{msec}$ ) \\
\hline Brainstem auditory evoked potentials & Evoked on $\geq 1$ side (latency of $\mathrm{V}$ wave may be delayed up to $1 \mathrm{msec}$ ) \\
\hline Cortical auditory evoked potentials & Evoked on $\geq 1$ side \\
\hline
\end{tabular}

Myoclonus Rating Scale ${ }^{7}$ (UMRS), electroencephalography, and visual and somatosensory evoked potentials with the stimulator on and with it off. After the evaluations, the independently obtained scores were compared, and any differences were jointly discussed. Differences in CRS-R scores by the 2 teams were rare, and the difference in the total scores of the 2 teams was never greater than 1 . Myoclonus was also evaluated by 2 independent teams before and after DBS surgery. After DBS, all the evaluators were aware that the subject had undergone surgery, but they did not know if the stimulator was on or off.

The study required that, at the end of the 1st year of thalamic DBS, the legal representative of the patient formally request that the stimulation be continued or that it be stopped. If the representative requested that the stimulation be stopped, he or she also had to decide between simple inactivation of the stimulator or removal of all the implanted devices.

\section{Neuroradiological Examinations}

Preoperative and postoperative MRI of each patient's brain was performed with a 1.5-T apparatus. For each patient, T1-weighted images (obtained with and without contrast) and T2-weighted images were acquired volumetrically and used for all the neuroanatomical evaluations. Volumes were calculated after identification of the regions of interest with the help of OsiriX software (Pixmeo).

\section{Surgical Procedure}

After the induction of anesthesia in the patient, the
CRW stereotactic head frame (Integra-Radionics) was positioned, and the patient then underwent intravenous contrast-enhanced MRI and CT. After fusion of the MRI with the CT scan, the images were registered to an electronic version of the Schaltenbrand and Wahren stereotactic atlas (Integra-Radionics). Because of brain atrophy and alterations peculiar to each patient that resulted from traumatic brain injury, the atlas data had to be complemented with electrophysiological indications. We targeted the anterior intralaminar thalamic nuclei and adjacent paralaminar regions ${ }^{26}$ starting from a bur hole $(5 \mathrm{~mm}$ in diameter) located in the ipsilateral parietal bone. Five microelectrodes (microTargeting Model 22670, FHC Bowdoinham), which were $2 \mathrm{~mm}$ apart from each other, in 2 patients and $4 \mathrm{mi}-$ croelectrodes in 1 patient were guided to the target using the Ben Gun tool with 5 parallel channels.

The electrodes were advanced in 1-mm steps from 15 $\mathrm{mm}$ before the target to $10 \mathrm{~mm}$ after it. After recording was done, the microelectrodes were removed, and a definitive quadripolar electrode (Model 3387, Medtronic Italia S.p.A.) was positioned with the second contact from the tip corresponding to the target position. The definitive electrodes were externalized through a disposable connecting cable for neurophysiological monitoring and testing during the first postoperative week. After that period, with the patient in a state of general anesthesia, the electrodes were connected with extension cables to a Kinetra pulse generator (Model 7428, Medtronic Italia S.p.A.) implanted in the right thoracic wall. The position of the electrodes relative to the thalamus was confirmed by postoperative CT scanning and MRI (Fig. 1).

TABLE 4. Neuroradiological inclusion criteria

\begin{tabular}{ll}
\hline \multicolumn{1}{c}{ Location } & $\begin{array}{c}\text { Criteria } \\
\text { Frontal }\end{array}$ \\
\hline Temporal & $\begin{array}{c}\text { Absence of damage to the posterior two-thirds of the left or dominant temporal lobe; extent of damage in the remaining structures of } \\
\text { the temporal lobes should be }<70 \% \text { of the total volume of the lobes }\end{array}$ \\
\hline Parietal & Damaged volume in $\geq 1$ parietal lobe should be $<30 \%$ \\
\hline Occipital & Damaged volume in $\geq 1$ occipital lobe should be $<30 \%$; the visual cortex should be spared in $\geq 1$ lobe \\
\hline Hypothalamus & Absence of lesions \\
\hline Thalamus & $\begin{array}{l}\text { Bilateral absence of damage to the centromedian } / \text { parafascicularis complex; the remaining structures of the thalami should not be } \\
\text { damaged }>40 \% \text { of the total volume of the } 2 \text { thalami }\end{array}$ \\
\hline Mesencephalon & Absence of damage in the region located between the substantia nigra \& the bases of the colliculi \\
\hline Pons & Absence of damage unless unilateral \& limited to the ventral third of the pons \\
\hline Cerebellum & Absence of lesions $>80 \%$ of the total volume; the deep nuclei of $\geq 1$ side of the cerebellum should be intact \\
\hline Medulla & Absence of damage unless limited to 1 pyramid \\
\hline Cervical cord & Absence of damage \\
\hline
\end{tabular}



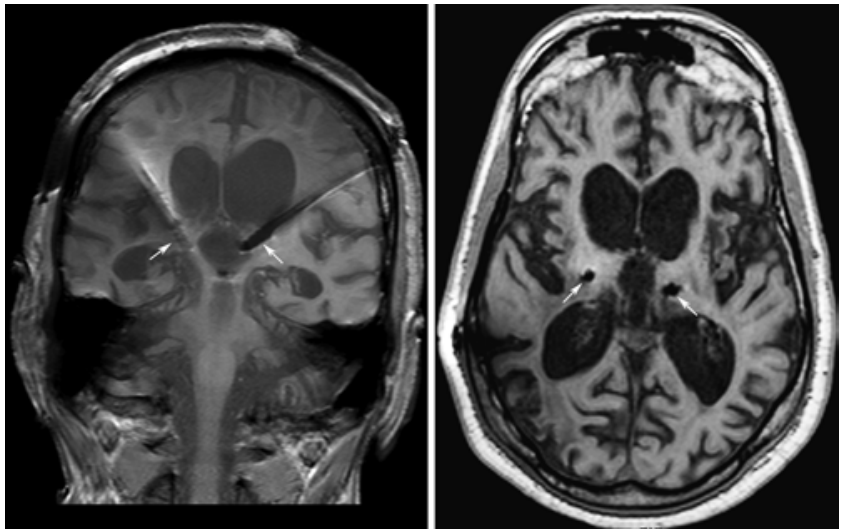

FIG. 1. Patient 1. T1-weighted MR images obtained after bilateral implantation of the stimulating electrodes in the central thalamus. The white arrows indicate the positions of the electrode leads. Left: Coronal image. The appearance of the DBS electrodes and their accompanying artifacts in the 2 sides differ because of slight differences in their anteroposterior position. The definitive positions of the electrodes were selected during surgery by using the data on the prevalence of burst activity obtained in the initial microelectrode exploration of the target region. Right: Axial image of the same patient.

\section{Stimulation Parameters}

During the initial testing phase, which lasted for 7 days, a wide variety of combinations of electrode contacts and stimulation parameters, including a trial with low stimulation frequency (less than $40 \mathrm{~Hz}$ ), were tested through the exteriorized leads of the electrodes. The individualized results of the initial phase enabled us to choose the definitive stimulation parameters that were further optimized on the basis of the clinical and electrophysiological responses of the patients in the months after stimulator implantation.

\section{Assessment of the Patients by Caregivers and Relatives}

By interviewing and collecting all spontaneous declarations of the patients' caregivers and relatives, we systematically assessed the degree of psychological discomfort induced in them by the surgical procedures and the following period of DBS stimulation in the patients. Using the same methods, we also evaluated the temporal evolution of their assessment of the degree of worsening or improvement that DBS induced in the patients' status and in their ability to manage them.

\section{Neurophysiology}

Multichannel EEG (SystemPLUS, Micromed) recordings were obtained before and after surgery with a sampling rate of $2048 \mathrm{~Hz}$ and an analog-to-digital converter resolution of 16 bits with a standard montage (16 electrodes). Auditory, visual, and somatosensory evoked potentials were recorded using standard stimuli and the same apparatus used for EEG recordings.

EEG signals were first filtered in the frequency range $(1-40 \mathrm{~Hz})$ in which we found approximately $99 \%$ of the signal power spectrum and then further parted by a finite impulse response filter $(0.3-\mathrm{Hz}$ width) in the 5 bands: delta (1-4 Hz), theta (4-8 Hz), alpha (8-13 Hz), beta (14-30 Hz), and low gamma $(30-40 \mathrm{~Hz})$. The fast Fourier transform
EEG power spectrum was estimated by the spectopo.m function of EEGLAB, an open-source toolbox for the analysis of single-trial EEG dynamics. ${ }^{6}$

\section{Statistical Analysis}

Statistical analyses and graphic rendering were performed with the help of MedCalc version 14.8.1.

\section{Patients}

\section{Patient 1}

Eight years before the implantation of DBS electrodes into the thalami, this 29-year-old man was involved in a car accident in which he suffered a closed head injury. The patient immediately lost consciousness and never regained it; findings on the initial CT and MR images were compatible with diffuse axonal injury. During the initial management, an external ventricular drain was positioned in the right lateral ventricle. After the initial trauma, the patient never regained consciousness and remained institutionalized in a VS/UWS. He was able to breathe without mechanical support through a tracheostomy; his cardiovascular, respiratory, renal, and gastrointestinal functions were normal. He had generalized spasticity with bilateral myoclonic mass movements. Opsoclonic and myoclonic movements could be evoked or exaggerated by minor tactile stimulation or environmental sounds (startle myoclonus); spontaneous bilateral opsoclonus was also often present and persisted for hours once it started. The CRS$\mathrm{R}$ score at the time of electrode implantation was 6 . The follow-up period was 59 months.

\section{Patient 2}

Two years and 10 months before the implantation of DBS electrodes into the thalami, this 23-year-old man was involved in a car accident that resulted in bilateral frontal acute subdural hematoma associated with multiple parenchymal lacerations. On the same day of the injury, the patient underwent surgery for evacuation of the hematomas and decompressive craniectomy. Four months after the initial surgery, he underwent cranioplasty with autologous bone. After the initial trauma, he never regained consciousness and remained institutionalized in a VS/UWS. He was able to breathe, and his cardiovascular, respiratory, renal, and gastrointestinal functions were normal. He had generalized spasticity and evoked myoclonus. The CRS$\mathrm{R}$ score at the time of electrode implantation was 8 . The follow-up period was 60 months.

\section{Patient 3}

Two years and 4 months before the implantation of DBS electrodes into the thalami, this 58-year-old man was involved in a car accident that resulted in multiple lacerations and contusions in frontal lobes bilaterally and the right parietal lobe; a subarachnoid hemorrhage was also present. He was treated conservatively and was never submitted to neurosurgical treatment with the exception of a right frontal intraparenchymal catheter being placed for measuring his intracranial pressure. After the initial trauma, he never regained consciousness, although he showed initial improvement from VS/UWS to MCS (he 
sometimes moved his right hand on request). He was institutionalized after discharge from the hospital in which he was initially treated. His CRS-R score at the time of electrode implantation was 14 . The follow-up period was 18 months, at the end of which all the implanted devices were removed because of a scalp infection, as described below.

\section{Results}

\section{Study Participants}

Of 40 patients evaluated for entry into the study, 14 had had a traumatic event, 20 had had an initial hypoxic/ ischemic insult caused by cardiac arrest, and 6 had had a spontaneous intracerebral hemorrhage; only 5 patients met all the eligibility criteria, and 3 underwent bilateral thalamic electrode implantation. The most common cause of exclusion was the presence of anatomical damage of the brain that exceeded the criteria listed for the study. Of the 2 patients who met all the eligibility criteria but did not proceed to undergo surgery, 1 exited the study because his legal representative chose to give priority to orthopedic surgery, which caused an excessive delay, and there were unexpected problems in the determination of the legal representative of the other patient.

\section{Clinical Outcomes}

After individualized clinical trials with a combination of stimulation parameters, we found that, for all patients, high-frequency stimulation (median $100 \mathrm{~Hz}$, minimum $80 \mathrm{~Hz}$, maximum $110 \mathrm{~Hz}$ ) was more efficient than lowfrequency stimulation $(<40 \mathrm{~Hz})$ in improving the patients' status and decreasing unwanted effects (e.g., spasms, persistent fear expression). Two patients had more adverse effects related to unipolar stimulation and therefore were chronically stimulated in bipolar mode, whereas 1 patient was stimulated in unipolar mode. Stimulation ended at 9:00 PM and restarted early in the morning at 7:00 AM. After the start of DBS, the average ( \pm SD) CRS-R scores improved from $1.67 \pm 1.53$ after 6 months of stimulation to 2 \pm 1.53 after 12 months and to $2.33 \pm 1.15$ after 18 months . For the 2 patients who continued DBS after 18 months, the average improvement in CRS-R scores from the preoperative score at 4 months was $3 \pm 0.00$ and remained stable up to 4 years.

Another favorable result of the stimulation was the large improvement in the severity and frequency of myoclonus, opsoclonus, and spasticity that we observed in all the patients; the larger effects were seen in the patients in a VS/UWS (Patients 1 and 2). Myoclonus was assessed by using the sum of the scores (range $0-125$ ) of Sections 2 (rest subscore range 0-108) and 3 (stimulus sensitivity subscore range $0-17$ ) of the $\mathrm{UMRS}^{7}$ (Fig. 2 right). The reduction in the resting myoclonus subscores in the 2 patients who had this problem before electrode implantation was remarkable, whereas their stimulus-evoked myoclonic movements were less affected. Effects on spasticity and myoclonus largely depended on ongoing stimulation, as shown by the reversal of the beneficial action of stimulation on resting myoclonus 1 hour after the stimulation was stopped. The beneficial effects of stimulation on spasticity did not revert completely when the stimulator was off, because muscle tone remained less than that before DBS when tested by passive movements.

Apart from their initial stress related to the electrode implantation, which quickly resolved, the caregivers and relatives were always relieved by the positive effects of the thalamic stimulation on spasticity and myoclonus and by their own impression of an improved response to behavioral stimulation. Moreover, at the end of the first year of stimulation when, according to the protocol, the
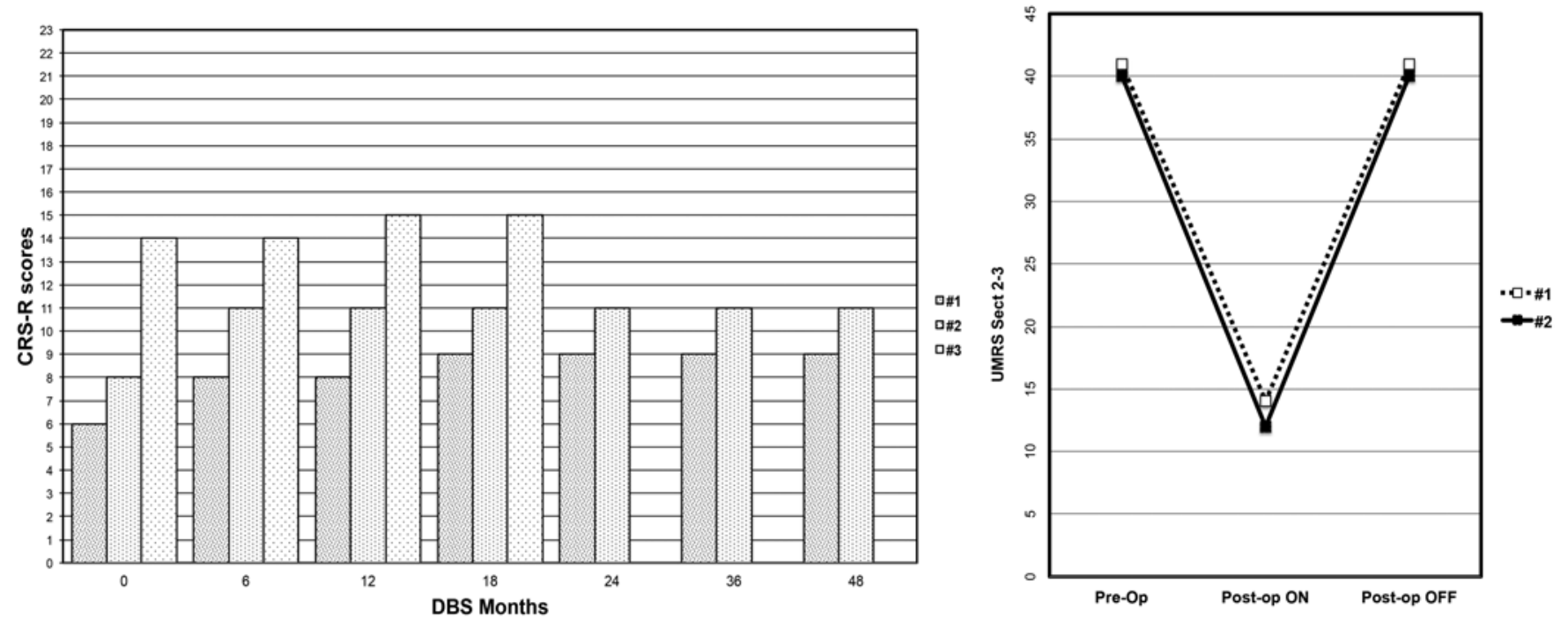

FIG. 2. Results of stimulation of the central thalamus during 4 years, as exemplified by the variations in CRS-R and UMRS scores. Left: Histogram showing the CRS-R scores before (time 0) and after DBS; stimulation was stopped in Patient 318 months after DBS implantation. Right: Individual (patients 1 and 2) myoclonus scores before and 6 months after implantation of DBS electrodes. The postoperative scores were calculated with the stimulator on or 2 hours after the stimulator had been turned off. Because of the DOC affecting the patients, assessment of myoclonus severity was limited to Sections 2 (rest) and 3 (stimulus sensitivity) of the UMRS. 
legal representatives had to decide whether to halt DBS, all of them independently opted for continuing the treatment even though none of the patients had regained full consciousness.

\section{Neurophysiological Outcomes}

Visual, acoustic, and somatosensory (N20/P25 bilateral median nerve stimulation) evoked potentials did not change significantly after DBS. After 1 month of stimulation, the EEG power spectrum showed increased power at the theta and gamma frequencies (Fig. 3). Overall, the EEG spectral power increased in all patients and for all bands except for the alpha and beta bands in the patient in an MCS (Fig. 4). These changes were stable and depended on DBS stimulation. We obtained further indications that DBS modulated the EEG activity of the patients by independent component analysis (ICA). We computed the power of ICA components before and after DBS with the stimulator on or off. We consistently found that preDBS and post-DBS component power distributions on the scalp were remarkably similar when the stimulator was off, whereas significant differences were observed with the stimulator on (Fig. 5).

\section{Complications}

The surgery was well tolerated by all 3 patients. The initial CT images of the brain, which were required by pro- tocol within 12 hours after implantation of the electrodes, showed an intraparenchymal hematoma in the right parietal lobe, close but not concentric to one of the electrode tracks, in Patient 3 (Fig. 6). Considering the lack of significant mass effect and the stability of the clinical features of the patient, we treated the hematoma conservatively and did not remove the electrode. There were no perioperative infections. Seventeen months after implantation of the DBS electrodes, Patient 3 developed a scalp infection (Pseudomonas aeruginosa) with multiple small ulcers that caused limited exteriorization of one of the electrocatheter extensions. The infection started from a small superficial wound and presumably resulted from excessively vigorous combing during daily care. After a limited trial of intravenous antibiotic treatment (1 g of meropenem 3 times/ day), we discussed the situation with the patient's family and the person who was legally responsible for him, who finally decided for surgical removal of all the devices implanted for DBS. The surgery to remove the hardware was uneventful, and the infection resolved after 1 more week of antibiotic therapy.

\section{Discussion}

The results of our prospective multiinstitutional trial involving patients in a VS/UWS or MCS suggest that bilateral stimulation of the anterior intralaminar thalamic nuclei and adjacent paralaminar regions improves the clinical
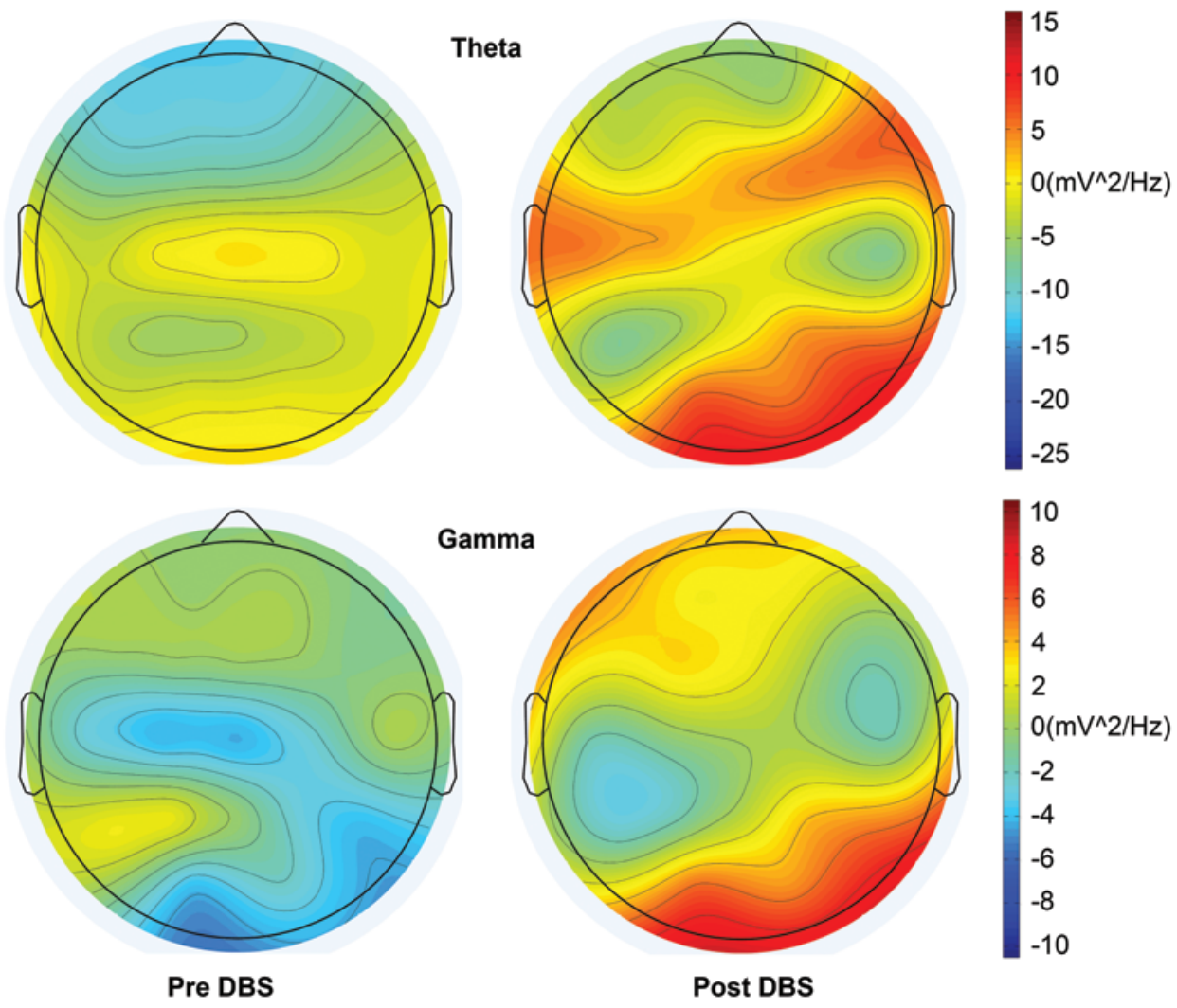

FIG. 3. Spectral heat maps (calculated by fast Fourier transform) representing the power spectrum of the theta and gamma activity in the scalp electroencephalogram recorded from patient 1 before (Pre DBS) and after (Post DBS) implantation of the electrodes. After 1 month of stimulation, the maps changed significantly, with an increase in power in both frequency bands. Left: Before DBS electrode implantation. Right: One month after DBS electrode implantation. 

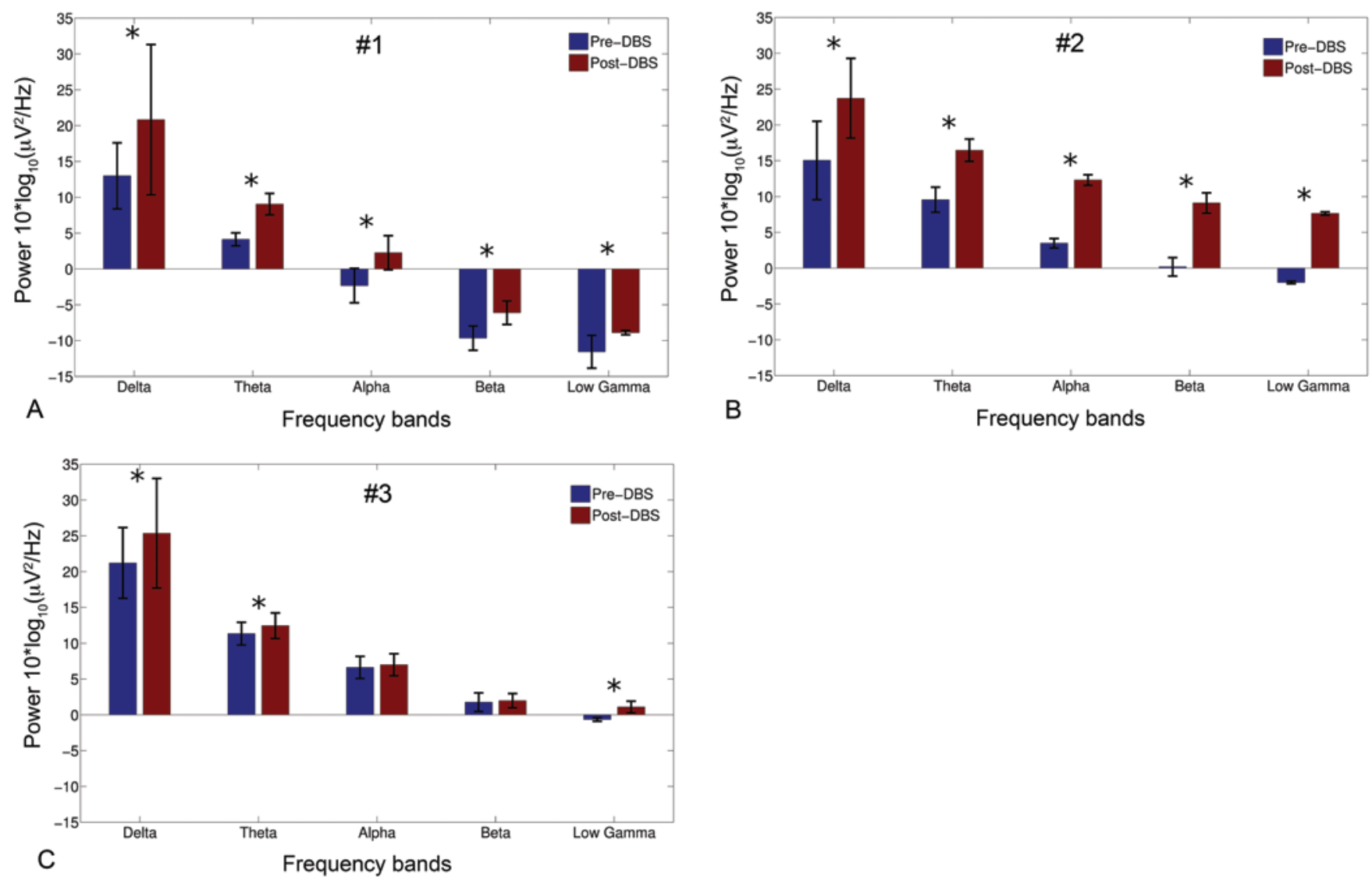

FIG. 4. Histograms displaying the power spectral analysis of the EEG recordings of the 3 patients collected before (blue) and 6 months after (red) activation of thalamic DBS. A: Patient 1 (VS/UWS). B: Patient 2 (VS/UWS). C: Patient 3 (MCS). The histograms clearly show a conspicuous increment of EEG spectral power in each patient and for each band except for the alpha and beta bands in the MCS patient for whom no statistical differences emerged (alpha, $p=0.310$; and beta, $p=0.647$, Bonferroni corrected nonparametric Wilcoxon rank-sum test). Asterisks indicate that the difference between the 2 recording conditions reached statistical significance. Figure is available in color online only.

status of the patients treated. In our experience, clinical improvement occurred in the patients in a VS/UWS and in the patient in an MCS despite the initial injuries having occurred more than 2 years (median 34 months, minimum 28 months, maximum 96 months) before DBS. Spontaneous improvements in patients with a severe DOC of posttraumatic origin happen mostly within 2 years after the initial injury. ${ }^{23,28,30}$ Thus, the improvements in the CRS-R scores seen in our patients are unlikely to have been spontaneous. The beneficial effects on spasticity and myoclonus were also a significant part of the clinical improvement. These effects were directly related to stimulation and decayed rapidly after turning off the stimulator. Thalamic DBS has already been described for the treatment of myoclonus caused by perinatal anoxia ${ }^{16}$ and in myoclonusdystonia syndrome. ${ }^{12,17}$ Thalamic electrodes for the treatment of myoclonus are usually positioned into the ventral intermediate nucleus of the thalamus, ${ }^{25}$ which is located far from our target even when taking into account the relative shrinkage and distortion of the thalamus commonly seen in patients with a DOC. However, there are indications that stimulation of the centromedian-parafascicularis nucleus may also modulate motor output. ${ }^{2}$ Previous studies with electrodes implanted unilaterally or bilaterally in the thalamus have resulted in a generalized increase in EEG frequency and desynchronization;;,32 these changes, however, were not necessarily predictive of recovery of consciousness. ${ }^{5,32}$ In our experience, bilateral thalamic stimulation induced statistically significant increments of the EEG spectral power in all patients and for all frequency bands with the exception of the alpha and beta bands in the patient in an MCS. We obtained further indications that stimulation induced changes in cortical activity by ICA ${ }^{34}$ of the EEG activity recorded before and during DBS. In all patients, the activation maps obtained with the stimulator off were similar to those obtained before DBS implantation, but they differed when the stimulator was on. Initial studies that used DBS in patients with a DOC were performed before the identification of $\mathrm{MCS}^{8}$ as a condition that is substantially different from VS/UWS..$^{18}$ It was suggested more recently that only patients in the upper end of an MCS could benefit from thalamic DBS. ${ }^{26}$ This opinion is not supported by either our results, which show that 2 patients in a VS/UWS for more than 2 years had improvements in their status and moved from a VS/UWS to an MCS after DBS, or the data from previous studies. ${ }^{3,32,33}$ However, previous data that supported positive effects of DBS in patients in a VS/UWS were obtained from patients 

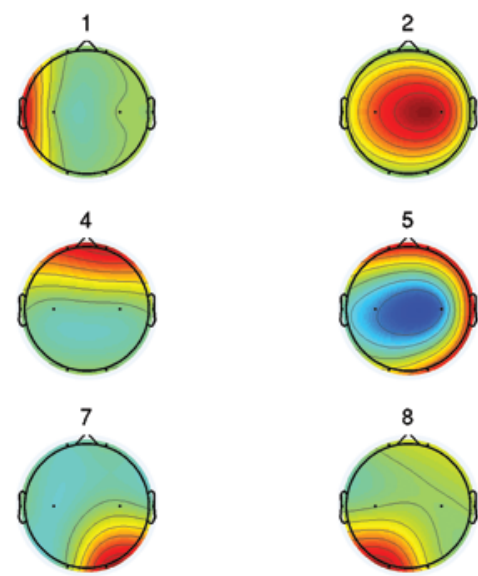

A
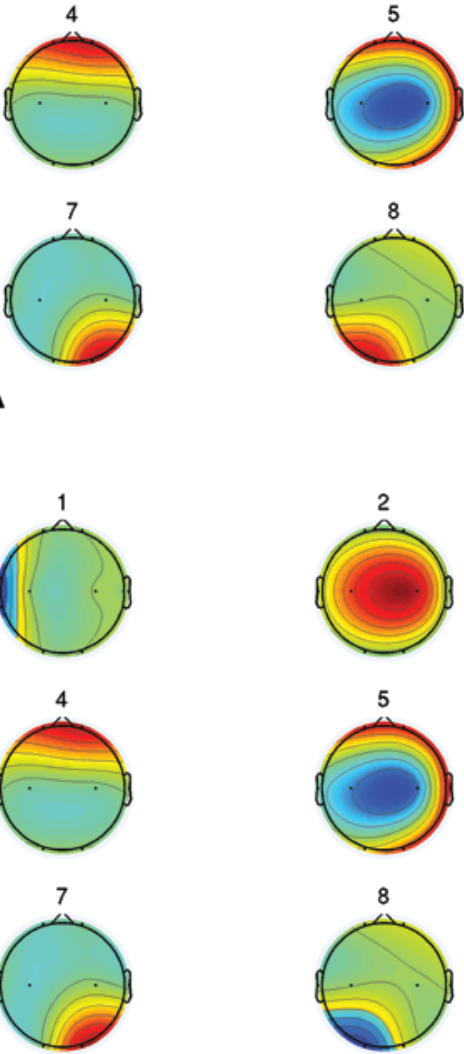

C
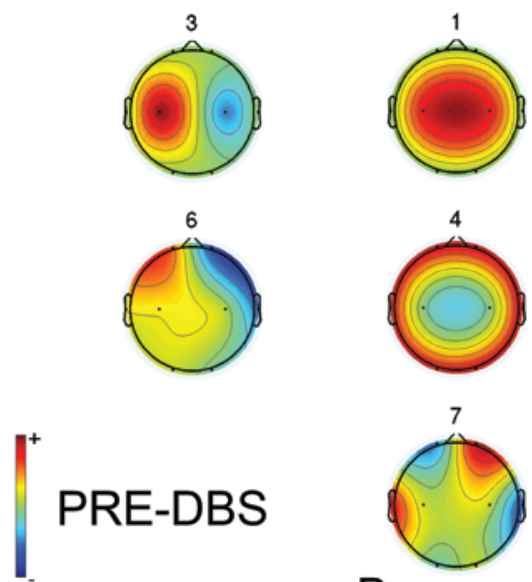

PRE-DBS
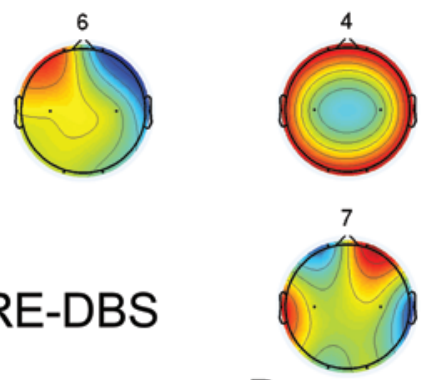

B
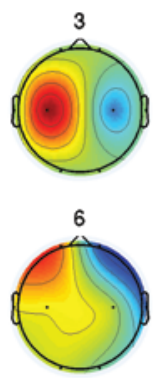

\section{DBS OFF}

FIG. 5. Activation maps resulting from EEG data analysis using time-domain ICA (Patient 2). Power distribution maps obtained before implantation of thalamic electrodes $(A)$ were significantly different than those obtained after electrode implantation with the stimulation on (B). However, maps obtained with the stimulator off $(\mathbf{C})$ were very similar to those obtained from the same patient before implantation of the DBS electrodes.

treated by DBS after only 3-8 months of stable VS/UWS, a period too short to rule out spontaneous recovery. ${ }^{26,28}$ As demonstrated by MRI and postmortem studies, overall structural brain integrity is less affected in patients in an MCS than in those in a VS/UWS. ${ }^{1}$ However, functional MRI (fMRI) studies performed to evaluate preserved awareness in the absence of reliable communication found that some patients in a VS/UWS, and some patients in an MCS, had specific brain-activation patterns reflecting awareness and cognition. ${ }^{21,22}$

In our study, the patient in an MCS had improvements in CRS-R scores but did not reach consistent communicative behavior; the same was true for the 2 patients in a VS/UWS. The difference between our results and those of Schiff et al. ${ }^{26}$ may be at least partially due to the difference in the level of MCS, because our patient did not mouth single words on prompting. The intracerebral hematoma located close to the track of the right stimulating electrode is unlikely to have been the cause of the incomplete results in our patient in an MCS, because the baseline CRS-R score (determined before electrode implantation) remained the same after complete resolution of the hematoma. In conclusion, our study results suggest that stimulation of the thalamus in patients with a DOC improves the reactivity of the patients to the environment and decreases spasticity and myoclonic movements. The patients' caregivers and relatives had favorable opinions of DBS during the entire first year and continued to remain in favor of it in the following years, even though the patients never reached consistent, explicitly communicative behavior.

Our study also had limitations. Five patients entered the study, and only 3 patients finally underwent thalamic DBS. However, our 3 patients, who were accrued for the study within a 2-year period, should be considered in the light of recent publications that were either based on a single patien ${ }^{26}$ or, even if they involved a higher number of patients, were not prospective and had accrual periods longer than 10 years. ${ }^{3,32}$ With overly protracted accrual periods, significant changes in hardware and sometimes even in the targets of the stimulation can occur. ${ }^{32}$ All these confounding factors limit the advantage of the increased numerosity of the sample. Our inclusion and exclusion criteria did 


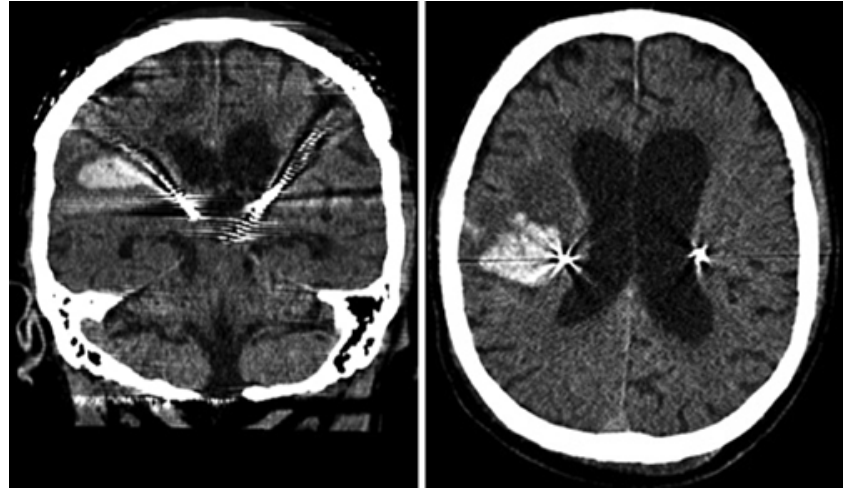

FIG. 6. Patient 3. Coronal (left) and axial (right) CT images obtained 12 hours after bilateral implantation of DBS electrodes in the central thalamus. The clinical status of the patient was unchanged, and the CT scan was obtained as a protocol-prescribed routine. An intraparenchymal hematoma, with maximum anteroposterior and transverse diameters of 33 and $28 \mathrm{~mm}$, respectively, was present just lateral to the course of the right stimulating electrode, at the level of the white matter of the right parietal lobe; additional blood, presumably from the electrode track, was visible in 1 sulcus. The hematoma resolved spontaneously and did not interfere with the stimulation protocol.

not incorporate fMRI results, which may have helped to distinguish among patients with a DOC and those with a higher potential to respond to thalamic DBS. ${ }^{24}$ However, our study was designed in 2007, before the publication of any study clearly demonstrating the clinical relevance of fMRI in the assessment of patient ability to generate willful responses during mental imagery tasks. ${ }^{22,29}$ Moreover, our study was open to all patients with a DOC, and prediction of the clinical course of such patients by fMRI seems to be less accurate in patients in an MCS than in those in a VS/UWS. ${ }^{29}$

The inclusion/exclusion criteria of our study reflected our goal of including patients in a VS/UWS and those in an MCS, provided their status was stable and there were no doubts in assigning them to one or the other group. As a minimal requirement for thalamic DBS, we excluded patients with obvious lesions of the thalami as assessed by MRI and/or bilateral lack of sensory evoked potentials (somatosensory and visual). We also excluded all patients with large lesions affecting the dominant hemisphere or both the frontal lobes, which are invariably associated with severe neurological disorders (e.g., global aphasia) that could abolish the benefits of any potential improvement in self-awareness. ${ }^{11}$ Despite our minimalistic exclusion criteria, more than $87.5 \%$ of the patients initially evaluated as potential candidates were excluded because MRI proved anatomical damage of the brain that exceeded the admission criteria.

\section{Conclusions}

Our study results suggest that the majority of patients with a DOC do not meet the minimal criteria of brain connectivity and tissue preservation that are required for attempting thalamic DBS to improve their condition. Patients with a DOC who met the minimal criteria and proceeded to undergo DBS experienced objective improvements in their condition, as shown by increases in their
CRS-R scores. However, we should consider that the leading motivation for society to sustain the cost of DBS in patients with a DOC should be restoration of consistent communicative behavior. Based on our experience and the results of more than 50 years of previous attempts, this is still an unlikely result of thalamic DBS in patients with a DOC.

\section{Acknowledgments}

We thank Drs. Ilaria Chiaranda and Hector Pesantez Orellana for help during the initial surgical procedures. Special thanks go to Roberto Scarselli, Simone Brasolin, and Lorena Bellini for their help in the operating room and to Mauro Tachimiri together with all the EEG technicians for their help with intraoperative recordings.

The study was supported by a grant (ricerca corrente 08019701/08) to Drs. Imberti and Magrassi from the Fondazione I.R.C.C.S. Policlinico S. Matteo - Pavia. Funding source had no role in study design and implementation.

\section{References}

1. Adams JH, Jennett B, Murray LS, Teasdale GM, Gennarelli TA, Graham DI: Neuropathological findings in disabled survivors of a head injury. J Neurotrauma 28:701-709, 2011

2. Caparros-Lefebvre D, Blond S, Feltin MP, Pollak P, Benabid AL: Improvement of levodopa induced dyskinesias by thalamic deep brain stimulation is related to slight variation in electrode placement: possible involvement of the centre median and parafascicularis complex. J Neurol Neurosurg Psychiatry 67:308-314, 1999

3. Cohadon F, Richer E: [Deep cerebral stimulation in patients with post-traumatic vegetative state. 25 cases.] Neurochirurgie 39:281-292, 1993 (Fr)

4. Cohadon F, Richer E, Rougier A, Deliac PH, Loiseau H: Deep brain stimulation in cases of prolonged post-traumatic unconsciousness, in Lazorthes Y, Upton ARM (eds): Neurostimulation: An Overview. Mt Kisco, NY: Futura Publishing Company, 1985

5. Deliac P, Richer E, Berthomieu J, Paty J, Cohadon F, Bensch C: [Electrophysiological development under thalamic stimulation of post-traumatic persistent vegetative states. Apropos of 25 cases.] Neurochirurgie 39:293-303, 1993 (Fr)

6. Delorme A, Makeig S: EEGLAB: an open source toolbox for analysis of single-trial EEG dynamics including independent component analysis. J Neurosci Methods 134:9-21, 2004

7. Frucht SJ, Leurgans SE, Hallett M, Fahn S: The Unified Myoclonus Rating Scale. Adv Neurol 89:361-376, 2002

8. Giacino JT, Ashwal S, Childs N, Cranford R, Jennett B, Katz DI, et al: The minimally conscious state: definition and diagnostic criteria. Neurology 58:349-353, 2002

9. Giacino JT, Kalmar K, Whyte J: The JFK Coma Recovery Scale-Revised: measurement characteristics and diagnostic utility. Arch Phys Med Rehabil 85:2020-2029, 2004

10. Giacino JT, Whyte J, Bagiella E, Kalmar K, Childs N, Khademi A, et al: Placebo-controlled trial of amantadine for severe traumatic brain injury. N Engl J Med 366:819-826, 2012

11. Glannon W: Neurostimulation and the minimally conscious state. Bioethics 22:337-345, 2008

12. Gruber D, Kühn AA, Schoenecker T, Kivi A, Trottenberg T, Hoffmann KT, et al: Pallidal and thalamic deep brain stimulation in myoclonus-dystonia. Mov Disord 25:1733-1743, 2010

13. Hassler R, Ore GD, Bricolo A, Dieckmann G, Dolce G: EEG and clinical arousal induced by bilateral long-term stimulation of pallidal systems in traumatic vigil coma. Electroencephalogr Clin Neurophysiol 27:689-690, 1969 
14. Hassler R, Ore GD, Dieckmann G, Bricolo A, Dolce G: Behavioural and EEG arousal induced by stimulation of unspecific projection systems in a patient with post-traumatic apallic syndrome. Electroencephalogr Clin Neurophysiol 27:306-310, 1969

15. Kalmar K, Giacino JT: The JFK Coma Recovery Scale-Revised. Neuropsychol Rehabil 15:454-460, 2005

16. Kobayashi K, Katayama Y, Otaka T, Obuchi T, Kano T, Nagaoka T, et al: Thalamic deep brain stimulation for the treatment of action myoclonus caused by perinatal anoxia. Stereotact Funct Neurosurg 88:259-263, 2010

17. Kuncel AM, Turner DA, Ozelius LJ, Greene PE, Grill WM, Stacy MA: Myoclonus and tremor response to thalamic deep brain stimulation parameters in a patient with inherited myoclonus-dystonia syndrome. Clin Neurol Neurosurg 111:303-306, 2009

18. Laureys S, Celesia GG, Cohadon F, Lavrijsen J, LeónCarrión J, Sannita WG, et al: Unresponsive wakefulness syndrome: a new name for the vegetative state or apallic syndrome. BMC Med 8:68, 2010

19. Lemaire JJ, Sontheimer A, Nezzar H, Pontier B, Luauté J, Roche B, et al: Electrical modulation of neuronal networks in brain-injured patients with disorders of consciousness: a systematic review. Ann Fr Anesth Reanim 33:88-97, 2014

20. Lombardi F, Gatta G, Sacco S, Muratori A, Carolei A: The Italian version of the Coma Recovery Scale-Revised (CRSR). Funct Neurol 22:47-61, 2007

21. Monti MM, Rosenberg M, Finoia P, Kamau E, Pickard JD, Owen AM: Thalamo-frontal connectivity mediates top-down cognitive functions in disorders of consciousness. Neurology 84:167-173, 2015

22. Monti MM, Vanhaudenhuyse A, Coleman MR, Boly M, Pickard JD, Tshibanda L, et al: Willful modulation of brain activity in disorders of consciousness. N Engl J Med 362:579-589, 2010

23. Nakase-Richardson R, Whyte J, Giacino JT, Pavawalla S, Barnett SD, Yablon SA, et al: Longitudinal outcome of patients with disordered consciousness in the NIDRR TBI Model Systems Programs. J Neurotrauma 29:59-65, 2012

24. Owen AM, Coleman MR, Boly M, Davis MH, Laureys S, Pickard JD: Detecting awareness in the vegetative state. Science 313:1402, 2006

25. Rughani AI, Lozano AM: Surgical treatment of myoclonus dystonia syndrome. Mov Disord 28:282-287, 2013

26. Schiff ND, Giacino JT, Kalmar K, Victor JD, Baker K, Gerber M, et al: Behavioural improvements with thalamic stimulation after severe traumatic brain injury. Nature 448:600603, 2007 (Erratum in Nature 452:120, 2008)

27. Sen AN, Campbell PG, Yadla S, Jallo J, Sharan AD: Deep brain stimulation in the management of disorders of consciousness: a review of physiology, previous reports, and ethical considerations. Neurosurg Focus 29(2):E14, 2010

28. Tsubokawa T, Yamamoto T, Katayama Y, Hirayama T, Maejima S, Moriya T: Deep-brain stimulation in a persistent vegetative state: follow-up results and criteria for selection of candidates. Brain Inj 4:315-327, 1990

29. Vogel D, Markl A, Yu T, Kotchoubey B, Lang S, Müller F: Can mental imagery functional magnetic resonance imaging predict recovery in patients with disorders of consciousness? Arch Phys Med Rehabil 94:1891-1898, 2013

30. Whyte J, Gosseries O, Chervoneva I, DiPasquale MC, Giacino J, Kalmar K, et al: Predictors of short-term outcome in brain-injured patients with disorders of consciousness. Prog Brain Res 177:63-72, 2009

31. Yamamoto T, Katayama Y, Kobayashi K, Kasai M, Oshima H, Fukaya C: DBS therapy for a persistent vegetative state: ten years follow-up results. Acta Neurochir Suppl 87:15-18, 2003

32. Yamamoto T, Katayama Y, Kobayashi K, Oshima H, Fukaya C, Tsubokawa T: Deep brain stimulation for the treatment of vegetative state. Eur J Neurosci 32:1145-1151, 2010

33. Yamamoto T, Kobayashi K, Kasai M, Oshima H, Fukaya C, Katayama Y: DBS therapy for the vegetative state and minimally conscious state. Acta Neurochir Suppl 93:101-104, 2005

34. Zhukov L, Weinstein D, Johnson C: Independent component analysis for EEG source localization. IEEE Eng Med Biol Mag 19:87-96, 2000

\section{Disclosures}

The authors report no conflict of interest concerning the materials or methods used in this study or the findings specified in this paper.

\section{Author Contributions}

Conception and design: Magrassi, Biella, Imberti. Acquisition of data: all authors. Analysis and interpretation of data: Magrassi, Zippo, Biella, Imberti. Drafting the article: Magrassi, Imberti. Critically revising the article: Magrassi, Maggioni, Pistarini, Di Perri. Reviewed submitted version of manuscript: Magrassi, Bastianello, Zippo, Iotti, Biella, Imberti. Approved the final version of the manuscript on behalf of all authors: Magrassi. Statistical analysis: Magrassi, Zippo. Study supervision: Magrassi, Imberti.

\section{Supplemental Information}

\section{Previous Presentations}

Portions of this work were presented in abstract form at the Congress of the European Society of Stereotactic and Functional Neurosurgery held in Athens, Greece, on September 22-25, 2010, and in Cascais, Portugal, on September 26-29, 2012.

\section{Correspondence}

Lorenzo Magrassi, Neurochirurgia, Dipartimento di Scienze Clinico-Chirurgiche, Diagnostiche e Pediatriche, University of Pavia, Fondazione IRCCS Policlinico S. Matteo, V.le Golgi 19, 27100

Pavia, Italy. email: lorenzo.magrassi@unipv.it. 